\title{
Networking in times of pandemic
}

\author{
The shift to virtual meetings has made networking harder, but it has also brought forth benefits to the scientific \\ community that should be embraced moving forward.
}

t has been more than a year since the COVID-19 pandemic started, and the scientific community has had to quickly adapt in various ways to avoid the transmission of the virus and to keep everyone safe. Working from home became a necessity, and everything from classes to workshops, meetings and conferences have been mostly happening virtually. Videoconference applications have become essential tools for all of us, both in our professional and personal lives.

What has this meant for networking?

Networking is invaluable to researchers. Through networking, opinions are shared, ideas are developed, and collaborations are created. Conferences and workshops are the ideal environments for networking, since people from all around the world, from multiple research groups and sharing similar interests, get together in the same location to talk about science. The recent shift from in-person to virtual conferences, albeit vital for the safety of the scientific community, has created some challenges: while researchers can easily interact with each other when attending in-person events (remember coffee breaks?), the same does not naturally occur during virtual events. These barriers to networking can be particularly disadvantageous for new graduate students, or even for postdoctoral and early-career researchers, who tend to use conferences and workshops to meet new researchers, discuss their work, and make connections, all of which facilitates academic growth.

It is worth noting that the virtual platforms used in conferences have become incredibly sophisticated, even sometimes including virtual rooms for networking, which the research community can certainly appreciate. Nevertheless, it is hard to virtually replicate an environment that can lead to multiple, separate unexpected conversations at any time. Furthermore, potential time zone differences, and the fact that researchers are still working from home while attending virtual conferences, can further complicate their engagement in these events.

But every cloud has a silver lining. While the lack of networking opportunities in virtual settings is a disadvantage, the ability to attend conferences virtually increases accessibility. Virtual conferences reach broader audiences than their in-person counterparts, allow people to attend more meetings in a year, and make it cheaper for researchers from regions and universities that lack sufficient travel funds under normal circumstances to attend these events. Departments and labs can also more easily invite researchers from all around the world to give talks or seminars. Finally, flying to conferences and meetings creates a large carbon footprint, which virtual meetings can help to substantially decrease ${ }^{1}$.

What will the future hold? As the COVID-19 vaccination efforts accelerate in multiple countries, in-person conferences will certainly start to come back. We argue that these conferences and events should still try to maintain a virtual component for those who cannot attend in person; as a matter of fact, scientists do want virtual meetings to stay after the pandemic, mainly due to the aforementioned advantages, even though they might impact networking ${ }^{2}$.

Until we can all meet together in a conference room, our tip is: don't be shy! After attending a virtual meeting, contact the researchers whose work interests you, schedule video calls with them, and most importantly, keep the conversation going. And if you are on the receiving end of these outreach inquiries, especially from younger researchers, please be mindful that this is one of the few ways we can network at the moment, and interact with your fellow researchers whenever possible.

Hopefully very soon, we will be able to network in person again. And as we get back to business as usual, let us not forget the lessons that we have learned regarding the benefits of promoting inclusion, expanding accessibility and striving to create a better environment for all of us.

Published online: 24 June 2021

https://doi.org/10.1038/s43588-021-00096-X

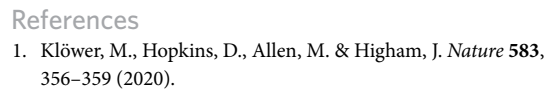

